\title{
FINNLAND UND FINNISCHE LITERATUR IN POLEN
}

\author{
BOLESŁAW MROZEWICZ \\ Adam Mickiewicz University in Poznań
}

ABSTRACT. The article describes the presence of Finland in the consciousness of Poles (since 14th-16th century) as well as the presence of the Finnish literature on the Polish publishing market since 1860s until today. Finnish literature became broadly known in Europe only in 1835, when the first edition of the national epic Kalevala appeared and directed European intelligentsia's attention to Finnish nation and its struggle for their own national identity. Selection of Finnish literary works (including those written in Swedish) which were translated in that times into Polish proves that the Polish publishers and translators were consciously looking for similarities between the situation of Poland and Finland which both were under the reign of tsarist Russia. The rich Finnish literature of the next decades fully shows the difficult process of becoming a Finn and finding one's own identity under new political and social conditions.

\section{ALLER ANFANG FREMDER KULTUREN IST SCHWER...}

Es ist nicht einfach zu Beginn des 21.Jhs., uns Polen, eindeutig zu sagen, wann eigentlich kulturelle und politische Beziehungen zwischen Finnland und Polen begonnen haben. Erst in der 2.Hälfte des 14. und im Verlauf des 15.Jhs. drangen nach Polen erste Berichte und Nachrichten über das Leben der Einwohner von Finnland. Ihre Verfasser waren hauptsächlich Danziger Kaufleute. Weiteren Informationen über dieses damals für Polen entlegene Land können wir für die Renaissancezeit begegnen. Der polnische Historiker, Geograph, Arzt und Professor der JagiellonenUniversität in Krakau Maciej von Miechów (Miechowita - Matthias de Miechow), veröffentlichte im Jahre 1517 sein Werk Tractatus de duabus Sarmatiia Asiana et Europiiana et de contetis $i$ eis (Der Traktat über zwei Sarmatien: Asiatisches und Europäisches und was sich dort befindet). Vier 
Jahre später veröffentlichte er dasselbe Werk unter einem anderen Titel: Descriptio Sarmatiarum (1521) - er schildert darin Osteuropa und das Leben seiner Bewohner. Matthias weiß, dass es so etwas wie Finnland gibt, ein „Land geographisch östlich von Schweden gelegen, politisch und administrativ zu ihm gehörend“. 1535 wurde das Werk aus dem Lateinischen ins Polnische übersetzt. Zum ersten Mal erscheint darin die polnische Bezeichnung für das Land: „Finlandya“. Finnland wird auch im Buch des polnischen Adeligen Marcin Bielski (1495-1575) erwähnt, des Anhängers des Protestantismus. 1551 erschien sein enzyklopädisches Werk Chronik der ganzen Welt, wo er mehr über Skandinavien und auch über Finnland schreibt. Es sei „ein Land von tausend Seen mit einem rauhen Klima und einem wundervollen, reizenden Boden“. Über politische Angelegenheiten schrieb dagegen ein anderer Wissenschaftler, Vertreter des Bürgertums, Humanist, Diplomat und schließlich auch Bischof von Lidzbark Warmiński (dt. Heilsberg) Marcin Kromer in seinem Werk Historyja prawdziwa o przygodzie żatosnej ksiązęcia finlandzkiego Jana i królewny polskiej Katarzyny (Die wahre Geschichte des trübseligen Abenteuers des finnländischen Herzoges Johannes III. Wasa und der polnischen Prinzessin Katharina Jagiellonica) - anonym 1570. Die Literatur der nächsten Jahrhunderte bezeugt kein allzu reges Interesse der Polen an Finnland. Erst in der 2.Hälfte des 18.Jhs. veröffentlichte der Historiker und Geograph Karol Wyrwicz das Handbuch Geographie der neuesten Zeit etc. (1768), in dem er genau die Einwohner Nordskandinaviens Lapplands - vorstellt, die ,in kleinen, hölzernen, mit Leder bezogenen Hüttlein hausen ... Kein Obstbaum kann dort wegen der kalten Luft gedeihen“ (Wyrwicz 1768:43).

Erst im 19.Jh. dringen mehr umfangreiche und sichere Berichte über Finnland und seine Bewohner nach Polen - Finnland war weiterhin für Polens Bürger ein exotisches und weit gelegenes Land. Der damaligen polnischen Presse können wir entnehmen, das Finnland von zwei Nationen bevölkert war: von der schwedischen (ca. 0,5 Mio.) und finnischen (2,5 Mio.). Aus dem Zeitungsartikel Lud fiński i jego kultura (Das finnische Volk und seine Kultur) in Tygodnik Ilustrowany (Illustrierte Wochenschrift) (Włodek 1907:808-809) ${ }^{1}$ konnten die Polen erfahren, dass die Finnen zur finnisch-ugrischen Völkergruppe gezählt werden. Der Finne sei „dunkelhaarig, mit rundem Gesicht, hervorstechenden Backenknochen, eher stämmig als groß und schlank. Vom Charakter her eher wortkarg, verschlossen, melancholisch und nachdenklich“ (Włodek 1907:808). In Gesellschaft - besonders in Gegenwart

${ }^{1}$ Die Zeitschrift Tygodnik Ilustrowany wurde 1859 gegründet und setzte sich zum Ziel, das ganze polnische Geistes- und Künstlerleben darzustellen. Besonders stark vertreten war darin die Geschichtspublizistik, Kultur-, Literatur- und Sittengeschichte. Nicht selten wurden auch Beiträge veröffentlicht, die die Geschichte anderer Völker schilderten. Seltener beschäftigte sich die Schrift dagegen mit politischen Fragen. 
von Ausländern - höflich und gastfreundlich. Im weiteren können wir über die Entwicklung und wachsende Bedeutung der finnischen Sprache erfahren, über Verdienste Gabriel Porthans und Adolf Ivar Arwidssons auf diesem Gebiet, über die Finnische Literaturgesellschaft (1831), über die Entwicklung der finnischen Presse (1820 - nur eine finnischsprachige und 3 schwedischsprachige Zeitungen; aber 1860 bereits je 14 Titel, 1900 - 73 schwedisch- und 99 finnischsprachige Schriften). Das Wissen über Finnland des 19.Jhs. wäre wohl viel oberflächiger und unvollständiger gewesen, wenn man in der polnischen Presse das Bild des finnischen Bauern ausgelassen hätte. Diese Lücke schließt der Artikel Finlandya $i$ Finlandczycy (Finnland und die Finnländer) von Bohdan Kutyłowski (1906). Der finnische Bauer befasse sich im Sommer mit Fischfang und ,setze in kümmerlichen Booten in Schneestürmen sein Leben zahlreichen Gefahren aus" (Kutyłowski 1906:92$6)^{2}$. Der Fischer vom Sommer verwandle sich im Winter in einen Jäger. Dieser Beruf sei sehr gefährlich, es könne passieren, dass sich große Eisbrocken loslösen und Jäger auf Eisschollen ins Unbekannte getrieben werden. Es gebe auch Arbeiter, die sich mit Holzflößerei befassen, sie stehen auf runden Baumstämmen, die ungeschickt zu Flößen zusammengebunden seien und verlassen diese nicht seit der Baum gefällt wurde, bis er ins Meer gelange. Zuweilen könne es bis zu zwei Jahren dauern, denn die Flöße frieren für sechs oder acht Monate zu (Kutyłowski 1906:92-6). Aus dem Artikel können wir auch einiges über die ursprünglichen Sitten und Bräuche sowie über das patriarchalische Leben der Finnen erfahren. Ihre Häuser seien mit grellem Rot gestrichen und jedes Haus besitze sein eigenes Bad (Sauna) mit einem aus Steinen gebauten Herd. Beim Badnehmen schütten die Finnen das Wasser auf glührote Steine und wenn der Dampf aufkomme, schlagen sie sich mit Birkenzweigen. (...) Die Finnen vergessen auch nicht - trotz strenger Klimabedingungen - ihre Kinder zu bilden. „Manchmal wandert die Schule auf dem Schiff oder Skiern in die entferntesten Ortschaften" (Kutyłowski 1906:92-6). Das in diesem Artikel enthaltene Bild steht im krassen Widerspruch zur negativen Äußerung des polnischen Nationaldichters Adam Mickiewicz, der seinerzeit - enttäuscht darüber, dass finnische Soldaten an der Niederschlagung des polnischen Nationalaufstandes gegen das zaristische Russland von 1863 mitgewirkt haben - in Collage de France in Paris sagte: „der Finne ist mürrisch, elend, wie geschaffen für Joch oder Zerstörungswerk“ (Tretiak 1882:60).

${ }^{2}$ Die radikale Krakauer Studentenzeitschrift Ognisko (Feuerherd), in der Kutyłowski seinen Beitrag über Finnland veröffentlichte, arbeitete mit der ebenso radikalen Studentenorganisation Czytelnia Akademicka - (Akademischer Lesesaal) in Krakau und Lwow (1889-90) zusammen. 


\section{FENNICA IN DER POLNISCHEN PRESSE DES 19.JHS.}

Die Presse war im dreigeteilten Polen des 19. und zu Beginn des 20.Jhs. jenes Organ, wo kulturelle, gesellschaftliche und politische - aber auch literarische - Tendenzen aus Europa, darunter auch aus Skandinavien, vorgestellt werden konnten. Recht früh begegnen wir darin den Übersetzungen finnischer Literatur ${ }^{3}$. Bereits um die Mitte des 19.Jhs. können wir die ersten literarischen Proben der Übersetzertätigkeit feststellen. Es waren vor allem Übersetzungen von Volksliedern oder kurzen Erzählungen. Das gebotene Bild der finnischen Literatur war sehr bescheiden und stellte eigentlich nur zwei Autoren aus Finnland vor: Johan L. Runeberg und Elias Lönnrot. Die ersten Übertragungen Runebergschen Gedichte wurden in der für Polen im russischen Teilungsgebiet wichtigen Monatsschrift Biblioteka Warszawska (Warschauer Bibliothek) - bis 1876 die einzige wissenschaftliche Zeitschrift in Polen - veröffentlicht. Im Kapitel Pieśni ludu na pótnocy (Lieder der Völker im Norden) ist auch der Artikel Nowoczesne pieśni ludu finlandzkiego (Moderne Lieder des finnländischen Volkes) mit drei Liedern: Das Wiegenlied, Die Liebe und Der Bach in Übersetzung von Leon Potocki auf der französischen Vorlage Marmiiers zu finden (Runeberg 1853:320-321). Ebenfalls in dieser Zeitschriftnummer wurde im Artikel Pótnoc skandynawska $w$ ciagu ostatniego pót wieku (Skandinavischer Norden im letzten halben Jahrhundert) ein weiteres - diesmal Prosawerk von Runeberg Die Bäuerin veröffentlicht. Der Übersetzer ist unbekannt. Im Artikel finden sich auch umfangreichere Informationen über die finnische Literatur. Der Autor spricht von der sog. finnischen literarischen Bewegung der Fennomannen, von der literarischen und philosophischen Zeitschrift Suomi und Suometar und ihrer Bedeutung für die Förderung der finnischen Sprache und Literatur. Im weiteren Teil beschäftigt er sich mit einigen finnischen Dichtern, z.B. Zachris Topelius und Fredrik Berndtson. Aus dieser kurzen Schilderung kann man sehen, dass das Interesse der Polen in dieser Zeit vor allem der Volksliteratur galt. Die Werke von Runeberg wurden in Polen auch in den 70er Jahren des 19.Jhs. gedruckt, und seine Erzählung Der Weihnachstabend in Finnland erschien in der literarischen Zeitschrift Kronika rodzinna 1868-1915 (Familienchronik). 1869 wurde auch dem polnischen Leser in Biblioteka Warszawska (Die Warschauer Bibliothek) zum ersten Mal das finnische Nationalepos Kalevala vorgestellt. Das Interesse für Kalevala war enorm, unabhängig davon, dass das Epos in Polen bisher wenig bekannt war - die

\footnotetext{
${ }^{3}$ Unter finnischer Literatur wird hier sowohl die schwedisch- als auch finnischsprachige Literatur verstanden. Die finnische Literatur wurde dem polnischen Leser anfangs vor allem Dank den Übersetzungen aus anderen Fremdsprachen - aus dem Französischen, Deutschen und Schwedischen - zugänglich gemacht.
} 
Übersetzungen des Werkes stützten sich vor allem auf westeuropäische, vor allem französische und deutsche Ausgaben. Im Artikel von Seweryna Duchińska finden wir nur eine sehr kurze Zusammenfassung des Inhalts und ein paar von ihr übertragene Fragmente, denen eine Einleitung vorangestellt wurde. Sie stellt Elias Lönnrot - den „finnischen Homer“ - und seine Verdienste um das Epos vor. „Er - der Runensammler - hätte seit 1828 jedes Städtchen, jedes Dorf, jede arme Dorfkate besucht. Ihm hätten auch arme Menschen aus Dankbarkeit für seine medizinischen Dienste Runen vorgesungen: selten singt Runoja ${ }^{4}$ allein. Meistens setzten sich zwei Männer gegenüber auf einem Brett (einer Bank) und im langsamen Schaukeln singen sie ein paar Stunden lang (...)“ (Duchińska 1869:245). Sie bespricht dann kurz die erste und die zweite Ausgabe des Werkes und ist bemüht, dem polnischen Leser den Inhalt des ganzen Werkes wiederzugeben.

In den letzten 25 Jahren des 19.Jhs. war das Interesse an der finnischen Literatur in Polen nicht allzu stark entwickelt (die Zeit des Positivismus) und es waren nur wenige finnische Texte auf polnisch erschienen. Und doch erfahren wir z.B. mehr über Johan L. Runeberg aus dem Artikel von Oskar Brzozowski Zapiski z pobytu w Szwecji (Notizen aus meinem Aufenthalt in Schweden) (Brzozowski 1878:459). Am Ende des Beitrags finden wir das Gedicht von einem alten Mann, der nach vielen Wanderjahren als Bettler in seine Heimat zurückkehrt, um dort zu sterben und zu einem besseren Leben zu erwachen. Mehr Aufmerksamkeit schenkte man der finnischen Novellenkunst, in der vor allem der finnische Alltag geschildert wurde. Hier seien realistische Novellen von Pietari Päivärinta Verfehltes Leben und In der Einsamkeit (Päivärinta 1894 - Übersetzer unbekannt) zu nennen. In der Literatur- und Kulturzeitschrift Przeglad Tygodniowy - 1866-1904 (Die Wochenschau) ist auch das Bild des alten finnischen Junggesellen in der Erzählung Der alte Junggeselle (übers. von Karol Bicz - eigentlich Ludwik Szczepański) enthalten. Die finnische Literatur in Polen war am Ende des 19.Jhs. insgesamt (auch in Bezug auf das Schaffen eines Autors) wenig und nur bruchstückhaft bekannt. Das Hauptinteresse galt vor allem dem Epos Kalevala - in der polnischen Presse war man bemüht, es immer wieder vorzustellen. Und so finden wir in der Warschauer Bibliothek erneut eine Besprechung des Werkes von Feliks Jeziorański - es war das erste kritische Studium zum Kalevala in Polen. Das Epos „schildere Ereignisse, Personen und Naturerscheinungen in einer schlichten, ruhigen Weise. Es zeichne sich durch seine unbeugsame Objektivität aus“" (Jeziorański 1880:230). Ein anderer Autor, der sich mit dem Epos in dieser Zeit befasste, war Historiker und Kenner der Romantik und der

\footnotetext{
${ }^{4}$ Duchińska leitet vom finnischen Wort „runo” (Gedicht) - völlig inkorrekt - den Namen für den Vortragenden ab. Im Finnischen benutzt man für den Volkssänger, der die „runot“ (Gedichte, Volkslieder) vorsingt, die Bezeichnung „laulaja“ (vom Verb: laulaa - singen). Duchińska (1869:240).
} 
romantischen Literatur in Europa Józef Tretiak - er veröffentlichte seine Beiträge zur Literatur und Kultur auf den Spalten des Przewodnik nankowy $i$ literacki - 1822 (Wissenschaftlich-literarischer Führer). In seiner Einleitung zum Werk schildert er, wie und warum dieses finnische Epos überhaupt entstand. Er schreibt u.a., Finnland hätte als Teil oder Provinz Schwedens seine eigene Volkspoesie nicht gekannt und auch nicht gesucht. Nach 1809 von Schweden losgelöst und Russland angeschlossen, hätten sich das Land und die Nation definieren müssen. Die Liebe der Finnen zu ihrer Heimat und die Hinwendung in der europäischen Literatur zum Volkstum hätte die finnische (zum großen Teil schwedisch sprechende Intelligenz) dazu angespornt, die eigene Volkspoesie zu sammeln und aufzuschreiben. Denn wenn Runoja singe, höre alles auf seine Lieder (Tretiak 1882:159-181). Tretiak zählt darin auch finnische Götter auf. Nach einer recht weitläufigen Einführung erzählt er den Inhalt des Werkes und stellt zum Schluss fest, dass Kalevala ein „fantastischer Traum sei“ (Tretiak 1882:261). Die Phantasie und Lyrismus seien - ihm nach - zwei hervorstechende Merkmale der finnischen Dichtung. „Der Charakter des finnischen Volkes, seine religiösen Vorstellungen, Sitten und Bräuche, gesellschaftliche Verhältnisse, all das wie es uns scheint - spiegele sich im finnischen Nationalepos wider" (Tretiak 1882:373). Ein gesteigertes Interesse für die finnische Literatur können wir in der neoromantischen Zeit in Polen beobachten. Große Verdienste um die Popularisierung dieser Literatur erwarb sich Gabriela Plewińska, die einen Abriss der ganzen finnischen Literatur Finlandia $w$ oświetleniu literackim. Zbiór utworów pisarzy finlandzkich (Finnland in literarischer Beleuchtung. Eine Sammlung von Werken finnischer Autoren) samt ausgewählten Erzählungen in der Serie Biblioteka Dziet Wyborowych (Bibliothek Auserkorener Werke) lieferte (Plewińska 1901). Auf dem historischen Hintergrund bespricht die Autorin jene Gestalten des literarischen Lebens in Finnland, die einen entscheidenden Einfluss auf die Entwicklung der finnischen Sprache und Literatur - und somit auch auf die Entstehung der finnischen Identität - hatten: u.a. das Schaffen von Elias Lönnrot, Johan L.Runeberg, Zachris Topelius, Juhani Aho, Minna Canth und anderen. Sie betont, dass die finnischen Autoren zu der Überzeugung gelangt seien, die finnische Literatur müsse national sein. Die Betonung des Finnischen und der finnischen Sprache und Kultur nimmt darin einen wichtigen Platz ein. Deshalb seien die Werke finnischer Autoren mit Landschafts- und Volksschilderungen überfüllt, z.B. bei Z.Topelius in seinem Gedicht Sonne des Nordens oder bei Adolf Paul in der Erzählung Legende der Wildnis; Runebergs Idyllen und Epigramme schildern häufig den Kampf der Bauern gegen die rauhe und unfreundliche Natur (Gedicht: Paavo aus Saarijärvi - P.Bonde). Sie betont auch, das finnische Volk befolge zahlreiche Traditionen und halte sich an das Alte fest, z.B. in den Romanen von Johan Jacob Ahrenberg Unser Landsmann 
(1897) oder Juhani Aho Ruhelos oder Der alte Mann vom See. Plewińska schildert auch die neue „moderne“ finnische Literatur, die sich in zwei Gruppen aufteile: in die Gruppe schwedisch sowie auch finnisch schreibender Schriftsteller. Die erste Gruppe werde von gebildeten Schriftstellern aus höheren Gesellschaftsklassen (Carl August Tavastjerna, Michael Lybeck Gabriel Lagus, Alexandra Gripenberg, Helena Westermarck, Adela Weman) und die andere von Schriftstellern und Schriftstellerinnen aus dem Mittelstand und niedrigeren Klassen vertreten. Als erste werden natürlich Minna Canth mit ihren zahlreichen Dramen und Juhani Aho als der bekannteste finnische Realist und Naturalist genannt. Zum Schluss befasst sich Plewińska mit jungen Lyrikern und Volksschriftstellern (Eino Leino, Pietari Päivärinta). Im zweiten Teil ihres literarischen Beitrages haben wir es mit Übersetzungen zu tun, u.a. der Erzählungen von Juhani Aho Der Alte vom See oder von Minna Canth Die alte Frau. Der von Plewińska erstellte Abriss der finnischen Literatur ist für die damalige Zeit sehr umfangreich und füllt fast im ganzen die beinahe 200 Seiten dicke Nummer der Reihe Biblioteka Dzieł Wyborowych (Bibliothek Auserkorener Werke). Außer dem vorgestellten Abriss konnte man in der polnischen Presse Übersetzungen vereinzelter Werke finnischer Autoren begegnen. So war es z.B. mit Erzählungen und Novellen von Juhani Aho Unter dem Pranger, Nach Helsinki (1889), Die Mutter oder von Pietari Päivärinta Der Reisebegleiter (Päivärinta 1907:379-416). Man konnte die Tendenz beobachten, dass das Interesse der polnischen Leserkreise an der finnischen Literatur im allgemeinen gestiegen war - dies war vor allem dem erwähnten Beitrag von Gabriela Plewińska zu verdanken.

\section{DIE ZEIT ZWISCHEN DEN WELTKRIEGEN (1918-1939)}

In der Zwischenkriegszeit konnte man ein kleines Tourismusaufkommen zwischen Polen und Finnland beobachten. Polnische Besucher waren von der Natur und Architektur beeindruckt und fasziniert. Ihre Berichte über Finnland waren damals von großer Bedeutung, denn sie erlaubten, das bescheidene Wissen über das Land im Norden Europas zu erweitern. Im Vordergrund standen literarische Reiseberichte der Schriftstellerin Maria Dąbrowska, (Dąbrowska 1913) die sich lebhaft für die Idee der Genossenschaften interessierte und deshalb mehrmals Reisen nach Finnland unternahm. Viel Platz räumte sie dem Stil der finnischen Architektur ein - finnische Dörfer seien entweder geschlossen oder in Verstreuung gebaut, zwischen den Wäldern und in der Wildnis, einsam verstreut (Dąbrowska 1913:13). Mehr Informationen über Finnland finden wir in ihrem Artikel A pótnoc zawsze mnie niezmiernie nęciła (Der Norden hat mich schon immer stark gelockt), (Dąbrowska 1974:435-439) in dem sie zugibt, dass sie anfangs vom Land und vor allem von ihren Bewohnern enttäuscht war: die Finnen seien zu langsam, 
und überall seien verschlossene Fenster zu sehen, alles sei irgendwie grau. Sie hat aber bald ihre Meinung geändert: an Stelle der Enttäuschung trat Begeisterung, vor allem für die Landschaft und das Klima. „In Polen ist der Sommer instabil, launisch. Hier in Finnland, wenn wir schon einen Sommer haben, so ist er mächtig - und so habe ich ihn erlebt. (...) Hier gibt es überall üppiges Grün - und wenn hier etwas duftet, so ist es einzig und allein der Nadelwald. Aber wenn er schon duftet, so duftet er grenzenlos, in ganzer Fülle, verschwenderisch“ (Dąbrowska 1974:437). Die Hauptstadt Helsinki ist in ihrer Schilderung wie „ein großer rauer Felsen“ (Dąbrowska 1974:438). Die Schönheit und die Anmut der Stadt liegen in ,ihrer schmucklosen Nacktheit darin seien ihre Redlichkeit und Natürlichkeit zu suchen“" (Dąbrowska 1974:439). Den Bemerkungen der polnischen Schriftstellerin ist insgesamt sehr viel Sympathie für dieses Land zu entnehmen.

Zahlreichen Reflexionen über Finnland begegnen wir in literarischen Reiseschilderungen in der Zeitschrift Przeglad Polsko-Fińsko-Estoński (Polnisch-Finnisch-Estnische Rundschau), die 1936 gegründet wurde. Sie setzte sich zum Ziel, ,intellektuelle, kulturelle und ökonomische Fragen in Polen, Finnland und Estland darzustellen und zu erörtern" (Piotrowski 1977:13-31). In dieser Zeitschrift schrieb die polnische Schriftstellerin Jadwiga Kunińska folgendermaßen über Finnland: „Die Schönheit der Stadt Helsinki - das ist Meer, moderne und originelle Architektur, Kunst- und Wissenschaftssammlungen; das sind schöne, saubere Strassen, Vorstadtgärten unter ruhig rauschenden Wäldern. Anmutig ist all das, was die Güte und Kultur dieser Menschen und der finnischen Erde spüren lässt" (Piotrowski 1977:13-31). Kazimiera Iłłakowiczówna - eine prominente polnische Schriftstellerin - schildert hingegen das Treffen mit einem Finnen, das ihr nicht aus dem Gedächtnis gehen wollte. Sie war fasziniert von seiner Schlichtheit, von der Wortkargheit, aber zugleich von der Einfachheit und Direktheit bei der Äußerung seiner Meinungen, von seiner Freundlichkeit. Jan Rostafiński, Professor an der Hochschule für Wirtschaft in Warschau, vergleicht in seinem Artikel Od Helsinek do Petsamo (Von Helsinki nach Petsamo) Finnland mit ,einem langen, breiten, rot gestrichenen Haus, mit weißen Fenster- und Türrahmen“ (Rostafiński 1937:7-10). Er spricht auch von den Bewohnern Lapplands, die ,in Häusern ohne Fenster, in meterhohen Pfahlbauten leben“. Die Berichte über Finnland aus dieser Zeit sind recht ergiebig und umfangreich. Aber auch wenn darin Schilderungen der Natur und Landschaft, sowie Architektur an Oberhand gewinnen, mangelt es auch nicht an anderen kulturellen Elementen und Berichten über die Finnen selbst.

In den Jahren 1918-1939 kann man bei dem polnischen Leser das wachsende Interesse für die finnische Prosa bemerken. Ein Beleg dafür sind recht häufige Übersetzungen von Werken finnischer Autoren. Und so kann man in der Halbmonatsschrift für Kinder und Jugend Ku stońcu (Der Sonne 
entgegen) von 1923 die Erzählung von J.W.Nylander Wintermorgen (übersetzt von Janina Mortkowiczowa) antreffen. Fragmente des Romans von Johannes Linnankoski Das Lied von der blutroten Blume wurde in der sozial-nationalen (sozialistischen) Wochenschrift Praca (Arbeit) veröffentlicht. Im Vorwort zum übersetzten Fragment (und zum Werk im allgemeinen) wurde die Haltung des Menschen gegenüber seiner Wirklichkeit hervorgehoben, sein Aufruhr gegen die bestehenden gesellschaftlichen Verhältnisse. In der Schrift Naokoto świata (Rund um die Welt) wurde im Jahre 1925 Larin-Kyöstis Erzählung Człowiek $i$ mech (Der Mensch und der Moos) in der Übertragung von Zofia de Bondy gedruckt. Sie erzählt vom Kampf des Menschen in der finnischen Einöde gegen Moos; scheinbar von dem Menschen besiegt, kommt er nach vielen Jahrhunderten - als er eines Tages bemerkt, dass der Mensch nicht da ist - zurück. „Wo ist der Mensch?“ - scheint hier die zentrale Frage zu sein. Der Mensch bemüht sich, dort, wo er gerade lebt, sich die Natur zu unterordnen. Der neue Mensch, der an Stelle des verstorbenen kommt, setzt den Kampf fort (Larin-Köysti 1925:222). Das Leben (und die Seele) des finnischen Volkes wird in meisterhafter, humorvoller Weise in KauppisHeikki's Erzählung Sasiedzi (Die Nachbarn) in der Zeitschrift Naokoło świata - 1927 (Rund um die Welt) geschildert. (Kauppis-Heikki 1927:159-166) 1933 wurde in Warschau eine Anthologie der Weltliteratur Panteon wielkich twórców poezji i prozy (Pantheon großer Dichter und Schriftsteller) von der Verlagsbücherei (Księgarnia Wydawnicza S.A.) herausgegeben. Viel Platz wurde darin - neben anderen Werken der Weltliteratur - der finnischen Prosa eingeräumt. Die Auswahl der Texte, die Struktur des Beitrages und Übersetzungen sind der Ethnographin Kazimiera Zawistowicz zu verdanken. Als erstes übersetzte sie die Erzählung von Zachris Topelius Brzoza $i$ gwiazda (Die Birke und der Stern), wo das Bild Finnlands vor 200 Jahren erscheint, als das Land die Hungerjahre erleben musste. „Man weiß nicht, wie und wo die Menschen starben und die Ehefrau wusste nicht, welches Schicksal ihrem Mann widerfahren war; der Bruder wusste nichts über ihren Bruder, Väter und Mütter wussten nicht, wohin sich ihre Kinder begaben und ob sie noch am Leben sind" (Topelius 1933:534-536). Unter den in der Zeitschrift vorgestellten Büchern durfte auch nicht das Fragment vom Aleksis Kivis Roman Sieben Brüder fehlen. Dasselbe betrifft das Werk von Juhani Aho - in die Anthologie wurde seine Erzählung Mój lud jałowcowi podobny (Mein Volk dem Wacholder ähnlich) aufgenommen, in der er die Frage stellt, warum der Finne seinen beharrlichen Kampf gegen die Natur nicht aufgibt. Ein weiteres Beispiel der finnischen Prosa in der Anthologie ist die Erzählung von Arvid Järnefelt $W$ pułapce (In der Falle) - der Verfasser vergleicht darin das Leben eines Mannes in der Familie mit der gefangenen Maus, die sich jetzt in der Falle befindet (Järnefelt 1933:544-545). Die Anthologie schließt ein Fragment aus der Erzählung von Johannes Linnankoski Zbiegowie (Die 
Flüchtlinge) ab. Der Autor greift auf die finnische Vergangenheit zurück und denkt über die Geschichte des Landes nach: „Wie viele Sorgen liegen in der finnischen Erde begraben. Wie viele Menschen haben in dieser eben Erde ihre großen und stillen Sorgen begraben“ (Linnankoski 1933:542-543).

Das Interesse der polnischen Literatur- und Übersetzerkreise an der finnischen Prosa verlief somit in zwei Richtungen. Übertragen wurden ins Polnische Werke der damaligen Gegenwartsautoren (Linnankoski, Larin Kyösti, Kaupis-Heikki, Nylander), aber man war gleichzeitig bemüht, die klassischen Werke der finnischen Literatur vorzustellen (Topelius, Kivi, Aho, Järnefelt), um auf die Wurzeln der finnischen Charakterzüge (sisu) - d.h. auf den Entstehungsprozess des finnischen Bewusstseins und der finnischen Identität - hinzuweisen. Große Verdienste um die Popularisierung der ganzen finnischen Literatur in dieser Zeit sind ohne Zweifel der Übersetzerin K. Zawistowicz zuzuschreiben, die die erwähnte Anthologie im Rahmen des Pantheons großer Dichter und Schriftsteller zusammenstellte. Beachtenswert ist derr Umstand, dass in Polen der Zwischenkriegszeit solche Schriftsteller wie F.E.Sillanpää, J.Lehtonen, I.Kianto, T.Pekkanen oder P.Haanpää ganz unbekannt blieben.

Im Bereich der finnischen Dichtung wurden weiterhin Gedichte von Johan L. Runeberg übersetzt, vor allem seine Liebeslyrik. Aber er war in Polen auch als finnischer Nationaldichter bekannt - deshalb war man bemüht, auf der Welle der von Polen unlängst (1918) erlangten Unabhängigkeit, die finnische Nationalhymne Nasz kraj (Unser Heimatland) zu übersetzen, um die Polen auf die Bedeutsamkeit der patriotischen Momente und der Liebe zum Vaterland, zur Landschaft des Landes und schließlich zur eigenen Heimat hinzuweisen (Runeberg 1921:4). Beliebt war in Polen auch die Dichtung von Eino Leino, der in seiner Lyrik viel Platz der Natur Kareliens und der Liebe gewidmet hatte. Wichtig für Polen war seine „Begeisterung“ für Polen - deshalb können wir in der Zeitschrift Przymierze (Das Bündnis) von 1921 sein Gedicht Es lebe Polen! (Vive la Pologne!) (Leino 1921:14) finden. Der Dichter drückt darin seine Freude aus, dass Polen seine Unabhängigkeit wieder gewinnen konnte. Er ruft auch zur Freundschaft zwischen Polen und Finnland auf. E.Leino schrieb auch poetische Prosa - zwei Beispiele aus seinem Zyklus $Z$ teki literackiej (Aus literarischer Theke) finden wir 1927 in der Zeitschrift Kobieta wspótczesna (Moderne Frau) (Leino 1927:15): es sind Morze i dzień (Das Meer und der Tag) und Porzadek (Die Ordnung). Insgesamt wurden in der Zwischenkriegszeit sieben Gedichte von E.Leino übersetzt.

Auch Gedichte von Antero Veikko Koskenniemi fanden Beachtung bei den Polen. 1917 verfasste er das Gedicht Runo vapaalle Puolallle, (Liryk na cześć wolnej Polski) (Csaplaros 1981:97) das mehrmals nach dem ersten Weltkrieg in Polen in verschiedenen Zeitungen gedruckt wurde, u.a. in Dziennik Polski (Polnisches Tagesblatt) in Petersburg am 29.April 1917, in 
Kurier Warszawski (Warschauer Kurier) 1918 oder in Legion Polski w Finlandii 1917-1927. (Die polnische Legion in Finnland 1917-1927) (Legion 1917-1921:1). Dieses Gedicht wurde zuerst während eines finnisch-polnischen Konzertes in Helsinki vorgetragen. Koskenniemi drückt darin seinen Wunsch aus, Polen möge ein freies Land für Ewigkeit bleiben. Dieselbe Freiheit wünscht er sich auch für Finnland. Außer hier genannten Dichtern wurden in den polnischen Zeitungen und Zeitschriften dieser Zeit noch andere Gedichte finnischer Autoren gedruckt. Fast alle - ähnlich wie andere finnische Werke wurden aus anderen Sprachen, vor allem aus dem Deutschen und Französischen übersetzt.

Das populärste Werk der finnischen Literatur in Polen der Zwischenkriegszeit bleibt aber ohne Zweifel das Epos Kalevala. Wie erwähnt, wurden erste Fragmente des Werkes (mit Erläuterungen) ins Polnische bereits 1869 von Seweryna Duchińska aus dem Französischen vollbracht. Nach 1918 kamen dann neue Impulse. In Przeglad Warszawski (Warschauer Rundschau) und Gazeta Literacka (Literarische Zeitung) wurden Fragmente des Kalevala in der Übertragung von Maria Krahelska (1925 - 1927) vorgestellt. Die Übersetzerin griff auf das Original zurück, stützte sich jedoch auf eine dänische Übersetzung. Zum hundertsten Jahrestag der Erstausgabe von Kalevala (1935) erschien in der Zeitung von Chełmno Kamienie (Steine) ein umfangreicher Beitrag von Sergiusz Kułakowski „Ludowy poemat fiński“ (Finnisches Volksgedicht) mit Runo 49, übersetzt von Kazimierz Andrzej Jaworski, der sich (erneut!) russischer Übersetzung bediente. Im gleichen Jahr stellte Jan Brzechwa in Kurier Poranny (Morgenkurier), Kurier Warszawski (Warschauer Kurier) und in Bluszcz (Der Efeu) Fragmente des finnischen Werkes vor. Weitere Teile des Werkes wurden erst nach dem 2.Weltkrieg in Twórczość -1946 (Das Schaffen), Odrodzenie - 1949 (Die Wiedergeburt) und im Band seiner Wiersze wybrane -1955 (Ausgewählte Gedichte) gedruckt. Dank den Veröffentlichungen in der Presse konnte das finnische Epos zu den breiten Bevölkerungsschichten gelangen und wurde immer beliebter, auch wenn das Wissen über Finnland, finnische Literatur und über das Kalevala selbst immer noch viel zu wünschen übrig ließ. Es fehlte immer noch an einer vollständigen Übersetzung aus dem finnischen Original.

\section{FINNISCHE LITERATUR IN POLEN NACH DEM 2.WELTKRIEG...}

Die ersten Jahre nach dem Zweiten Weltkrieg waren der Beschäftigung (und damit auch der Übersetzungsarbeit) mit der finnischen Literatur weniger hold. Erst gegen Ende der 50er Jahre kann man einen bescheidenen Aufbruch auf diesem Gebiet beobachten. Und wieder war hier die polnische Presse jenes Feld, auf dem einiges - was bisher unmöglich schien - doch möglich war. Das Tor zum Unmöglichen wurde in der Kinderliteratur aufgestoßen - in 
Kinderzeitschriften veröffentlichte man kleine Beiträge und Übersetzungen aus Fremdsprachen. 1958 bearbeitete Janina Porazińska für Kinder - in Form einer Erzählung - Motive aus dem Kalevala (dieses Datum wird auch als das Jahr der ersten polnischen Übersetzung des gesamten Werkes angesehen). 1967 wurde das Buch wiederaufgelegt. Es muss jedoch festgestellt werden, dass es keine „,buchstäbliche" Übersetzung war, sondern eine literarische Bearbeitung des Stoffes. Aus der Zeitperspektive muss man jedoch eingestehen, dass Porazińskas Fassung eine gelungene und zugleich eine wertvolle künstlerische Leistung ist, die auch heute zahlreiche junge Leser verlockt und dazu anspornt, mehr über finnische Literatur zu erfahren, sie zu suchen und zu lesen. Zum ersten Mal wurde der vollständige Text des Epos in Radar, einer in Polen sehr populären Jugendzeitschrift in den Jahren 1965-69 veröffentlicht, und zwar in der philologischen Bearbeitung von Karol Laszecki und künstlerischen Übersetzung von Józef Ozga-Michalski. Diese ZeitschriftFassung bildete dann die Grundlage für die Buchausgabe aus dem Jahre 1974 (762 Seiten) in Zusammenarbeit mit Suomalainen Kirjallisuuden Seura. Vor dem 150-Jubiläum der Erstausgabe des Kalevala (1998) wurde in der Übersetzung von Jerzy Litwiniuk die letzte polnische Ausgabe des Werkes veröffentlicht. Das Buch wurde außer Vor- und Nachwort mit einem recht großen Wörterbuch der Namen und Termini sowie der weniger bekannten polnischen Ausdrücke (hauptsächlich Archaismen, die die Übersetzung möglich gemacht haben) versehen. Außerdem konnte Litwiniuk aus dem Epos 54 verschiedene ,schamanistische“ Termini aussondern, die er dann zu einem geschlossenen Index zusammenstellte.

1974 wurde im Rahmen des Institutes für Germanistik der AdamMickiewicz Universität in Poznań Lehrstuhl für Skandinavistik gegründet 1975 nahm die Finnische Philologie die ersten Studenten auf. Auf Initiative der Mitarbeiter des Lehrstuhls begann man in Wydawnictwo Poznańskie (Posener Verlag) Werke skandinavischer - darunter auch finnischer - Autoren herauszugeben. In der Anfangsphase war man bemüht, dem polnischen Leser klassische Werke der finnischen Gegenwartsautoren vorzustellen, um u.a. all das nachzuholen, was man bisher versäumt hat. Da es in Polen damals im Bereich der Literatur noch kaum Übersetzer aus dem Finnischen gegeben hat, wurden die ersten Romane aus dem Deutschen (z.B. Frans E.Sillanpää) bzw. Schwedischen (Mika Waltari) übertragen. Die neue Generation der Übersetzter war erst im Kommen, und manche, die bisher deutsche Literatur übersetzt haben, beschlossen, Finnisch zu lernen (z.B. Cecylia Lewandowska). Nach und nach wurden im Verlauf der 70er und 80er Jahren Lücken geschlossen. Auf dem polnischen Markt konnte man immer häufiger Werken finnischer Autoren begegnen, die sich bei dem Leser großer Beliebtheit erfreuten. Und so wurden in diesen zwei Jahrzehnten in der Buchserie Skandinavischer Autoren Werke von Mika Waltari, Lauri Viita, Veijo Meri, Väinö Linna, Timo Mukka, 
Paavo Rintala, Paavo Haavikko, Hannu Salama, Toivo Pekkanen, Pentti Haanpää, Joel Lehtonen, Johannes Linnakoski, Eeva Joenpelto, Elvi Sinervo, Arvo Turtiainen herausgegeben. Bereits 1970 wurde vom Verlag Wydawnictwo Poznańskie die Anthologie Żyzny granit (Der fruchtbare Granit - 255 Seiten) veröffentlicht, die eine gute Einführung in die moderne finnische Kurzprosa darstellt. Außerdem wurde die Tradition fortgesetzt, Lyrik und kurze Prosaformen in den Zeitschriften zu drucken, um bei dieser Gelegenheit auch mehr (und schneller als im Verlagswesen) über Autoren und Literatur Finnlands berichten zu können. In der renommierten Reihe der Literaturzeitschrift Literatura na świecie (Literatur in der Welt) wurde auch gleich dreimal das dicke Heft (1977, 1988 und 1998) der finnischen Gegenwartsliteratur gewidmet. Große Verdienste erwarb sich auf diesem Gebiet die Übersetzerin aus der jüngeren Generation Joanna Trzcińska-Mejor, die außer Übersetzungsarbeit bemüht war - und immer noch ist - die finnische Literatur in Polen populär zu machen. Zu erwähnen ist auch die Monatsschrift Poezja (Dichtung), die 1982 ein Heft im Umfang von 126 Seiten ausschließlich der finnischen modernen Dichtung widmete. Es wurden dort Gedichte von Haavikko, Kirstinä, Kunnas, Manner, Melleri, Nieminen, Saarikoski, Saaritsa, Turtiainen vorgestellt. 1998 wurde - gewissermaßen als Fortsetzung dieser Anthologie - die Poezja fińska (Finnische Dichtung) herausgegeben, in der auch ältere Autoren wie Eino Leino oder Uuno Kailas mit ihren Gedichten vertreten sind. In den 90er Jahren des 20.Jhs. konnte man in Polen neue Auflagen von früher herausgegebenen und bei den Lesern beliebten finnischen Werken beobachten. Dazu gehört z.B. die Serie von den Mummins von Tove Jansson sowie zahlreiche (meistens historische) Werke von Mika Waltari, u.a. sein größter historischer Roman Sinhue der Ägypter. In dieser Zeit kamen auf den Markt auch neue Übersetzungen der Romane dieses Autors, u.a. Tajemnice Królestwa (Das Geheimnis des Königreiches) übersetzt von Kazimiera Manowska, Złotowłosa (Die Goldhaarige - J.Trzcińska-Mejor), Obcy przyszedt na farme (Der Fremde kam ins Haus); wie erwähnt wurde im Jahre 1998 erneut das Kalevala herausgegeben, dann zwei Bücher von Veikko Huovinen Hycler: życie $i$ dziatalność Hitlera (Hycler: Leben und Wirken Hitlers) und Wujaszek Józek (Onkelchen Josef) in Übersetzung von Kazimiera Manowska und Wspomnienia (Erinnerungen) von Carl Gustaf Mannerheim, übersetzt von Krystyna Szelągowska. In verschiedenen literarischen Zeitschriften - Magazyn Literacki, Dekada, Odra, Czas Kultury (Literarisches Magazin, Die Dekade, Die Oder, Kulturzeit) - konnte man Gedichte von Aaro Hellakoski, Sirkka Turkka oder Riina Katajavuori lesen. Nicht unbenannt bleiben sollte auch eine Anthologie der skandinavischen Kurzprosa Nie czytaj tej ksiażki (Du sollst dieses Buch nicht lesen - 1998); es ist eine Anthologie der von Studenten des Lehrstuhls für Skandinavistik in Poznań erstellten Texte 
skandinavischer Autoren. Einen wesentlichen Beitrag leisteten hier Studenten der Fennistik, die für den Band Texte von Leena Krohn übersetzt hatten.

\section{5. ÜBERSETZUNG FINNISCHER LITERATUR HEUTE...}

Im ersten Jahrzehnt des 21.Jhs. war die finnische Literatur in Polen vor allem dank der Tätigkeit zweier Verlage deutlich in Erscheinung getreten: es sind die Verlage Kojro und Słowo / obraz / terytoria (Wort / Bild /Territorien). In der Übersetzung von Bożena Kojro waren u.a. Listy do Trynidadu (Briefe nach Trinidad) von Annika Idström, Rozkaz (Der Befehl) von Leena Lander sowie Fantastyczne samobójstwo zbiorowe (Ein fantastischer Gruppenselbstmord) und Wyjacy mtynarz (Der heulende Müller) von Arto Paasilinna erschienen. Der zweite Verlag beschloss, in seiner Herausgebertätigkeit eine getrennte Buchreihe Terytoria Skandynawii (Territorien Skandinaviens) zu begründen. Die im Rahmen dieser Serie veröffentlichten Werke der finnischen Literatur wurden vor allem von Sebastian Musielak, dem Absolventen der Finnischen Philologie aus Poznań übersetzt. Er ist bemüht, den polnischen Leser u.a. mit den Romanen von Daniel Katz (Wie der Großvater auf Skiern nach Finnland kam), Joanna Sinisalo (Nicht vor Sonnenuntergang), Leena Lehtolainen (Die Schneefrau, Die Todesspirale) oder Sofi Oksanen (Das Fegefeuer) bekannt zu machen. Im Jahre 2008 wurde in der Zeitschrift Czas Kultury (Zeit für Kultur) die neueste finnische Prosa und Dichtung vorgestellt. Unter dem Motto „Finnisches Syndrom" kamen solche Autoren wie Veijo Meri, Markus Nummi, Tomi Kontio (Prosa) und Olli Heikkonen, Mikko Rimminen, Saila Susiluoto, Eino Santanen, Henrikka Tavi, Juhani Ahvenjärvi und Vilja-Tuulia Huotarinen (Lyrik) zu Wort. Außerdem wurde den präsentierten Texten eine recht umfangreiche Einführung in die neueste finnische Literatur vorangestellt. (Czas Kultury 2008:4-109) Im Bereich der Kinderliteratur (außer den Büchern über Die Mumins von Tove Jansson - hier: verschiedene Übersetzer im Verlauf der vergangenen 30 Jahre) - aktiv ist in den letzten Jahren Iwona Kosmowska-Kiuru, die die Kindebücher von Jari Mäkipää aus seiner Serie über den Klub Der Sturmwind (Huragan) übersetzt. Auch J. Trzcińska-Mejor übersetzt Kinderbücher, u.a. von Hannu Mäkelä Herra Huu (Pan Huczek 1998).

\section{SCHLUSSWORT}

Zusammenfassend sei festzustellen, dass die finnische Literatur in den letzten 40 Jahren in Polen verstärkt präsent ist. Sie ist vielfältig, erfasst verschiedene literarische Gattungen und Epochen und verschafft dem polnischen Leser die Möglichkeit, sich ein recht großes Wissen über Finnland 
und seine Bewohner anzueignen. Im Angebot steht nicht nur die schöngeistige Literatur - es gibt viele Sachbücher aus verschiedenen Gebieten, die ein kompaktes Bild dieses Landes liefern. Für den Leser wichtig bleibt jedoch die schön-geistige Literatur, die ihm die Möglichkeit eröffnet, die Seele (den Geist) - des ,historischen“, aber auch des „modernen“ Finnen - kennen zu lernen. Die Auswahl der Werke, die im 19. und in der ersten Hälfte des 20. Jahrhunderts aus dem Finnischen ins Polnische übertragen wurden, zeigt, dass für die Übersetzer die Entstehung der finnischen Identität eine große Rolle gespielt hat. Man verwies auf Parallelen in der politischen und gesellschaftlichen Situation zwischen Finnland und Polen, besonders bis 1918. Die Übersetzer haben - wie oft zu bemerken war - einen großen Einfluss auf die Wahl bestimmter Autoren und entsprechender Werke, die für das Panorama der finnischen Literatur wichtig sind. Dank ihrer Arbeit ist der polnische Leser ganz gut informiert, welche Themen in Finnland im 19. und 20.Jh. wichtig waren und was die Finnen im Zusammenhang damit geschrieben und gelesen haben und welche Literatur sie heute bevorzugen.

\section{LITERATUR}

Brzozowski, Oskar. 2008 [1878]. Zapiski z pobytu w Szwecji. [Kronika rodzinna, Serie: 3, 1878] Czas Kultury Nr. 2, S. 428-432.

Csaplaros, Istvan \& Joanna, Trzcińska-Mejor. 1981. Bibliografia literatury fińskiej w Polsce. Warszawa: Wydawnictwa Uniwersytetu Warszawskiego.

Dąbrowska, Maria. 1913. Finlandja - wzorowy kraj kooperacji. Warszawa: Drukarnia L. Bogusławskiego.

- 1974. A północ zawsze mnie niezmiernie nęciła. In: Ciesielski, Zenon (Hrsg.). Skandynawia w oczach Polaków. Gdańsk: Wydawnictwo Morskie, S. 435-439.

Duchińska, Seweryna. 1869. Epopeja finlandzka. Biblioteka Warszawska, Bd. III. S. 238-252.

Järnefelt, Arvid. 1933. W pułapce. In: Panteon wielkich twórców poezji i prozy. Warszawa Bd.2. S. 544-545.

Jeziorański, Feliks. 1880. Kalewala. Biblioteka Warszawska Bd. I. S. 29-51, 225-251.

Kauppis-Heikki. 1927. Sąsiedzi. Przekład z fińskiego. Naokoło świata Nr. 34, S.159-66.

Kutyłowski, Bohdan. 1906. Finlandya i Finlandczycy. Ognisko Nr. 2.

Larin-Köysti. 1925. Człowiek i mech. Übers. von Zofia de Bondy. Naokoło świata Nr. 16, S. 212-221.

Legion Polski w Finlandii 1917-1927. 1927. Warszawa: Nakładem Komisji Likwidacyjnej Legionu Polskiego w Finlandji z okazji dziesięciolecia (Broschüre).

Leino, Eino. 1921. Niech żyje Polska!. Übers. von Bohdan Kutyłowski. Przymierze Nr. 8, S.14.

- 1927. Z teki literackiej. Proza poetycka. (Morze i dzień, Porządek). Kobieta wspótczesna, Nr. 37, S. 15.

Linnankoski, Johannes. 1933. Zbiegowie. (Fragment). Übers. von K. Zawistowicz. In: Panteon wielkich twórców poezji i prozy. Warszawa Bd.2., S. 542-543.

Päivärinta, Pietari. 1892. Nowele fińskie (1: Chybione życie, 2: W samotni). Dziennik Łódzki Nr. 228-232, 234-236.

- 1907. Towarzysz podróży. Wiedza Bd. I., S. 379-384, 411-416.

Piotrowski, Bernard. 1977. Polsko-fińskie związki gospodarcze, kulturalne i naukowe w okresie międzywojennym. In: Komunikaty Instytutu Battyckiego, Gdańsk Heft 26. S. 13-31. 
Plewińska, Gabriela (Hrsg.) 1901. Finlandia w oświetleniu literackim. Zbiór utworów pisarzy finlandzkich. Übers. von Ernst Brauswetter (= Biblioteka Dziet Wyborowych Nr. 205).

Warszawa: Redakcya i Administracya Biblioteki Dzieł Wyborowych.

Rostafiński, Jan. 1937. Od Helsinek do Petsamo. Przegląd Polsko-Fińsko-Estoński Nr. 2.

Runeberg, Johan Ludvig 1853. Wieśniaczka. Übers. von Leon Potocki. Biblioteka Warszawska Bd. I. S. 320-321.

- 1921. Hymn finlandzki. Übers. von Bohdan Kutyłowski. Przymierze Nr.4.

Topelius, Zachariasz. 1933. Brzoza i gwiazda. In: Panteon wielkich twórców poezji i prozy. Warszawa. Bd.2, S. 539-540.

Tretiak, Józef. 1882. Kalewala. Epopeja fińska. Przewodnik Naukowy i Literacki. S. 60-71, 159-181, 261-282, 368-373.

Włodek, Ludwik. 1907. Lud fiński i jego kultura. Tygodnik Ilustrowany Nr. 40. S. 808-809.

Wyrwicz, Karol. 1773. Geografia powszechna czasów teraźniejszych. Warszawa: Coll. Soc. Jesu.

- 1768, 1770. Geografia czasów najnowszych. Warszawa: Komisja Edukacji Narodowej.

Zawistowicz, Kazimiera. 1932. Literatura fińska. In: Wielka Literatura Powszechna. Bd. 2, S. 29-737.

\section{Bolesław Mrozewicz}

Uniwersytet im. Adama Mickiewicza w Poznaniu

Instytut Językoznawstwa

Zakład Filologii Ugrofińskiej

Collegium Novum

al. Niepodległości 4

61-874 Poznań

Poland

bmrozewicz@gmail.com 